\title{
Dissection of the cecal microbial community in chickens after Eimeria tenella infection
}

\author{
Hong-Liang Chen ${ }^{\dagger}$, Xin-Yu Zhao ${ }^{\dagger}$, Guang-Xun Zhao, Hai-Bin Huang, Hao-Rui Li, Chun-Wei Shi, Wen-Tao Yang, \\ Yan-Long Jiang, Jian-Zhong Wang, Li-Ping Ye, Quan Zhao, Chun-Feng Wang ${ }^{*}$ and Gui-Lian Yang ${ }^{*}$ (])
}

\begin{abstract}
Background: Eimeria spp. are responsible for chicken coccidiosis which is the most important enteric protozoan disease resulting in tremendous economic losses in the poultry industry. Understanding the interaction between the avian cecal microbiota and coccidia is of interest in the development of alternative treatments that do not rely on chemotherapeutics and do not lead to drug resistance.

Methods: We utilized 165 rRNA gene sequencing to detect the dynamics of the cecal microbial community in AA broilers challenged with Eimeria tenella. Histopathological analysis of the cecum was also conducted.

Results: We found that microbial shifts occur during the infection. Lactobacillus, Faecalibacterium, Ruminococcaceae UCG-013, Romboutsia and Shuttleworthia decreased in abundance. However, the opportunistic pathogens Enterococcus and Streptococcus increased in abundance over time in response to the infection.

Conclusions: Eimeria tenella disrupts the integrity of the cecal microbiota and could promote the establishment and growth of potentially pathogenic bacteria. Defining bacterial populations affected by coccidial infection might help identify bacterial markers for intestinal disease as well as populations or species that could be beneficial in maintaining and restoring gut homeostasis during and after infection with E. tenella.
\end{abstract}

Keywords: Eimeria tenella, Chicken coccidiosis, Cecal microbiota, 165 rRNA, Alternative therapeutics

\section{Background}

Avian coccidiosis is the most important protozoan disease for the poultry industry worldwide resulting in substantial economic losses [1]. Seven species of apicomplexan parasites belonging to the genus Eimeria, i.e. E. tenella, E. necatrix, E. acervulina, E. maxima, E. brunetti, E. mitis and E. praecox, are the causative agents of coccidiosis [2]. The invasion of Eimeria specifically damages the intestinal epithelial cells and tissues of the caecum and disrupts gut homeostasis. Eimeria infection

\footnotetext{
*Correspondence: yangguilian@jlau.edu.cn; wangchunfeng@jlau.edu.cn; ${ }^{\dagger}$ Hong-Liang Chen and Xin-Yu Zhao contributed equally to this work College of Animal Science and Technology, Jilin Provincial Engineering Research Center of Animal Probiotics, Key Laboratory of Animal Production and Product Quality Safety of Ministry of Education, Jilin Agricultural University, Changchun, China
}

increases intestinal colonization of pathogenic bacteria, such as Clostridium perfringens [3], Salmonella enterica enterica serovar Enteritidis [4] and Campylobacter jejuni [5] and causes large shifts in microbial community structure [6-9]. Currently chemotherapeutic drugs are extensively utilized to control and prevent coccidiosis, but this has led to an unavoidable increase in drug resistance and drug residues, which raises public health concerns for poultry meat [10]. With the emergence of anticoccidial and antibiotic drug resistance, alternative strategies are urgently required to prevent the disease.

The gut microbiota is an "invisible organ" that has been discovered to play pivotal roles in host health [11]. The intestinal microbiota contributes to harvesting nutrition and energy from the diet, reducing adhesion of enteric pathogens, stimulating the proliferation of the gut epithelium and promoting the development 
of the immune system [12]. A growing body of research has focused on well-defined bacteria that play a vital role in the modulation of intestinal homeostasis. Probiotics are viable, well-defined bacteria that contribute to the health and balance of the intestinal tract and have the potential to provide protection against chicken coccidiosis [13-17]. Probiotic strains were assessed in vitro for anticoccidial activity to inhibit E. tenella sporozoite invasion into Madin-Darby bovine kidney (MDBK) cells [18]. Therapeutic intervention of gut microbiota is considered as a promising alternative measure to control coccidiosis in the future, although little is known about the interactions between gut microbiota and enteric protozoans.

The majority of previous studies on the enteric microbiota of Eimeria-infected chickens were conducted in vitro, relying on bacterial cultivation and counting [19]. With the development of next-generation sequencing technology, high-throughput sequencing methods have provided a more direct way to analyze microbial taxa in comparison to culture-dependent methods, thus the microbial composition can be better characterized [20]. MacDonald et al. [21] reported that E. tenella infection induced significant changes in the abundance of some microbial taxa with notable differences detected between lesion score categories, and severe pathology was associated with an increase of Enterobaceteriaceae and a decrease of Bacillales and Lactobacillales. Huang et al. [22] also found that perturbation of the microbiota was observed both in Arbor Acres (AA) broilers and White Leghorn chickens by $16 S$ rRNA sequencing during the oocyst shedding period in response to E. tenella infection, Clostridium and Escherichia increased, Lactobacillus and Faecalibacterium decreased. In the present study, our aim was to determine the changes in bacterial populations belonging to the cecal lumen in AA broilers using $16 S$ rRNA gene sequencing, to explore the dynamics of the microbiota associated with the different phases of $E$. tenella infection within an intact life-cycle.

\section{Methods}

\section{Chickens and parasites}

One-day-old AA broiler chickens were obtained from ShuangYang Broiler Hatchery (Jilin, China) and reared under coccidia-free conditions in flame sterilized wire cages. Feed and water were supplied ad libitum and no antibiotics or anticoccidial drugs were used. Chickens were randomly divided into four groups of 10 birds per group.

The E. tenella Beijing strain was utilized in this study. The oocysts were sporulated and purified according to the methods described in our previous studies $[23,24]$.

\section{Experimental design}

Forty AA broiler chickens with similar body weights were assigned equally into four groups: control group (C); merozoite reproduction group (M); gametocyte reproduction group $(\mathrm{G})$; and oocyst shedding group (O). The control group was a non-infected group, and the other three groups were E. tenella-infected groups. At 21 days of age, chickens in infected groups were inoculated with $5 \times 10^{4} \mathrm{E}$. tenella sporulated oocysts per chicken, while chickens in the control group were all sacrificed, the cecal contents of five birds were randomly selected and collected as fecal samples. At $105 \mathrm{~h}$ post-infection (hpi), all chickens in group $M$ were sacrificed, and the cecal contents of five individuals were randomly selected and collected as fecal samples. Then at $144 \mathrm{hpi}$ and $214 \mathrm{hpi}$, the stool samples of groups G and $\mathrm{O}$ were collected. All samples were stored at -80 ${ }^{\circ} \mathrm{C}$ before DNA extraction and sequencing. These four groups represent four phases of an intact life-cycle of $E$. tenella infection.

\section{Histological evaluation}

Cecal tissues from chickens from each group were excised, fixed in $10 \%$ formalin and embedded in paraffin wax. The tissues were dehydrated in a series of graded alcohols for staining and then sectioned. The sections were stained with hematoxylin and eosin (H\&E) and examined microscopically.

\section{DNA extraction, PCR amplification and 16S rRNA sequencing}

The metagenomic DNA was extracted from the samples utilizing the E.Z.N.A. ${ }^{\circledR}$ Stool DNA Kit (Omega Bio-Tek, Norcross, GA, USA) according to the manufacturer's instructions. The concentrations of the obtained DNA were determined by $1 \%$ agarose gel electrophoresis and spectrophotometry (optical density at $260 \mathrm{~nm} / 280 \mathrm{~nm}$ ratio). Then the V3-V4 hypervariable region of the $16 \mathrm{~S}$ rRNA gene was amplified by PCR. A pair of universal primers (338F: 5'-ACT CCT ACG GGA GGC AGC AG-3' and 806R: 5'-GAC TAC CVG GGT ATC TAA $\left.\mathrm{T}-3^{\prime}\right)$ were used [25]. These primers contained a set of 8-nucleotide barcode sequences unique to each sample. The PCR program involved an initial denaturation step at $94{ }^{\circ} \mathrm{C}$ for $3 \mathrm{~min}$, followed by 25 cycles of denaturation at $94{ }^{\circ} \mathrm{C}$ for $30 \mathrm{~s}$, annealing at $50{ }^{\circ} \mathrm{C}$ for $30 \mathrm{~s}$ and extension at $72{ }^{\circ} \mathrm{C}$ for $60 \mathrm{~s}$, with a final extension step at $72{ }^{\circ} \mathrm{C}$ for $7 \mathrm{~min}$. PCRs were performed in triplicate $25 \mu \mathrm{l}$ volumes containing $2.5 \mu \mathrm{l}$ of $10 \times$ Pyrobest Buffer $2 \mu \mathrm{l}$ (TaKaRa, Shiga, Japan), of $2.5 \mathrm{mM}$ dNTPs, $1 \mu \mathrm{l}$ of each primer $(10 \mu \mathrm{M}), 0.4 \mathrm{U}$ of Pyrobest DNA polymerase (TaKaRa), and $15 \mathrm{ng}$ of template DNA. 
PCR products were run in an electrophoresis chamber on a $2 \%$ agarose gel and purified using the AxyPrep DNA Gel Extraction Kit (Axygen Biosciences, Union City, CA, USA) following the manufacturer's instructions and quantified using QuantiFluor ${ }^{\mathrm{TM}}-\mathrm{ST}$ (Promega, Madison, Wisconsin, USA). Purified amplicons were used for library preparation and pyrosequencing. Sequencing libraries were generated using the NEBNext ${ }^{\circledR}$ Ultra $^{\mathrm{TM}}$ DNA Library Prep Kit (New England Biolabs, Ipswich, MA, USA) following the manufacturer's recommendations. Library quality was assessed and sequenced on an Illumina MiSeq platform PE300 platform (Illumina, Inc., CA, USA).

\section{Bioinformatics and sequencing data analysis}

The original DNA fragments were merged into tags using Trimmomatic (version 0.36; http://www.usade llab.org $/ \mathrm{cms} /$ ?page=trimmomatic) and FLASH (Fast Length Adjustment of Short reads, version 1.2.11; https ://ccb.jhu.edu/software/FLASH/) [26, 27]. Usearch (version 8.0.1623; https://www.drive5.com/usearch/) was applied to filter the chimeras and singletons of the raw sequencing data. Quality filtering of the raw tags was performed to generate high-quality clean tags according to Qiime (Quantitative Insights Into Microbial Ecology, v1.2.1; http://qiime.org/) [28]. Operational taxonomic units (OTUs) were clustered at 97\% sequence similarity following the Uclust (version 1.2.22; https://drive5.com/ usearch/manual/uclust_algo.html), and representative sequences of each cluster were used to assign taxonomy through annotation against the SILVA database. The alpha diversity of the samples, Chao 1 values, observed species and Shannon-Wiener indices were evaluated. Principal components analysis (PCA) of the OTUs in different groups was conducted using $\mathrm{R}$ version 3.5.1 Feather Spray (https://www.r-project.org/). Additionally, linear discriminant analysis (LDA) coupled with the effect size (LEfSe) algorithm (http://huttenhower.sph. harvard.edu/galaxy/) was conducted to identify the significant microbial differences among the groups [29]. The LDA score was calculated and a taxonomic cladogram was constructed to visualize the differences in microbial composition. A significance value of less than 0.05 and an LDA effect size of greater than 3 were used as thresholds for the LEfSe analysis. The raw reads were deposited into the NCBI Sequence Read Archive database (accession: SRP184532).

\section{Statistical analysis}

Comparisons between experimental groups were carried out using ANOVA followed by Tukey's honest significant differences (HSD) post-hoc test. All results are expressed as the mean \pm standard error (SE). $P$-values of $<0.05$ were considered significant.

\section{Results}

Histopathological analysis of the cecum

Microscopical examination showed that cecum glands were intact and no histopathological changes or necrosis were observed in the uninfected group (Fig. 1a). At 105 hpi, the structure of the cecum glands was indistinct and a large number of inflammatory cells were present in the submucosa (Fig. 1b). At $144 \mathrm{hpi}$, the intestinal glands and epithelial cells were invaded by coccidial gametocytes and oocysts. Inflammatory cells and villi blunting were also observed (Fig. 1c). At $214 \mathrm{hpi}$, several oocysts were observed in cecal glands and intestinal epithelial cells (Fig. 1d).

\section{Alpha and beta diversity of cecal microbial constitution after E. tenella infection}

The $16 S$ rRNA gene-based sequencing produced millions of raw reads. After assembly and filtration, a total of 1,053,731 sequences were obtained through MiSeq sequencing analysis from 20 samples, and the average length of the sequences was $419.16 \mathrm{bp}$. The Good's coverage index was greater than 99\%. In total, 1125 OTUs were observed in the four experimental groups. Good's coverage, the rarefaction, Shannon-Wiener, and OTU rank-abundance curves of all samples indicated that there was sufficient data sampling and adequate sequencing depth, and the database of $16 S$ rRNA gene sequences almost completely covered all microbial communities (Fig. 2).

The Chao 1 and observed species indices that estimate microbial richness, and the Shannon-Wiener index which measures species biodiversity, were calculated to evaluate the alpha diversity. Consideration of alpha diversity within the sequence datasets using the number of obtained OTUs, Chao 1, observed species and Shannon indices, showed no significant variation associated with E. tenella infection. There was no significant difference observed in the cecal microbiota alpha diversity between the control group and the infected groups. However, compared with the microbial diversity of the control group, the microbial biodiversity of the other groups increased after E. tenella infection. According to the Chao 1 index and observed species, we found that E. tenella infection led to an increase at the time point of $105 \mathrm{hpi}$ in group M, then a slight decrease at the time point of $144 \mathrm{hpi}$. In group $\mathrm{G}$, at the same time points, the Shannon-Wiener index indicated a similar trend. The microbial richness index in group $\mathrm{O}$ was the largest, and the Shannon-Wiener index of this group was lower than that of the other 

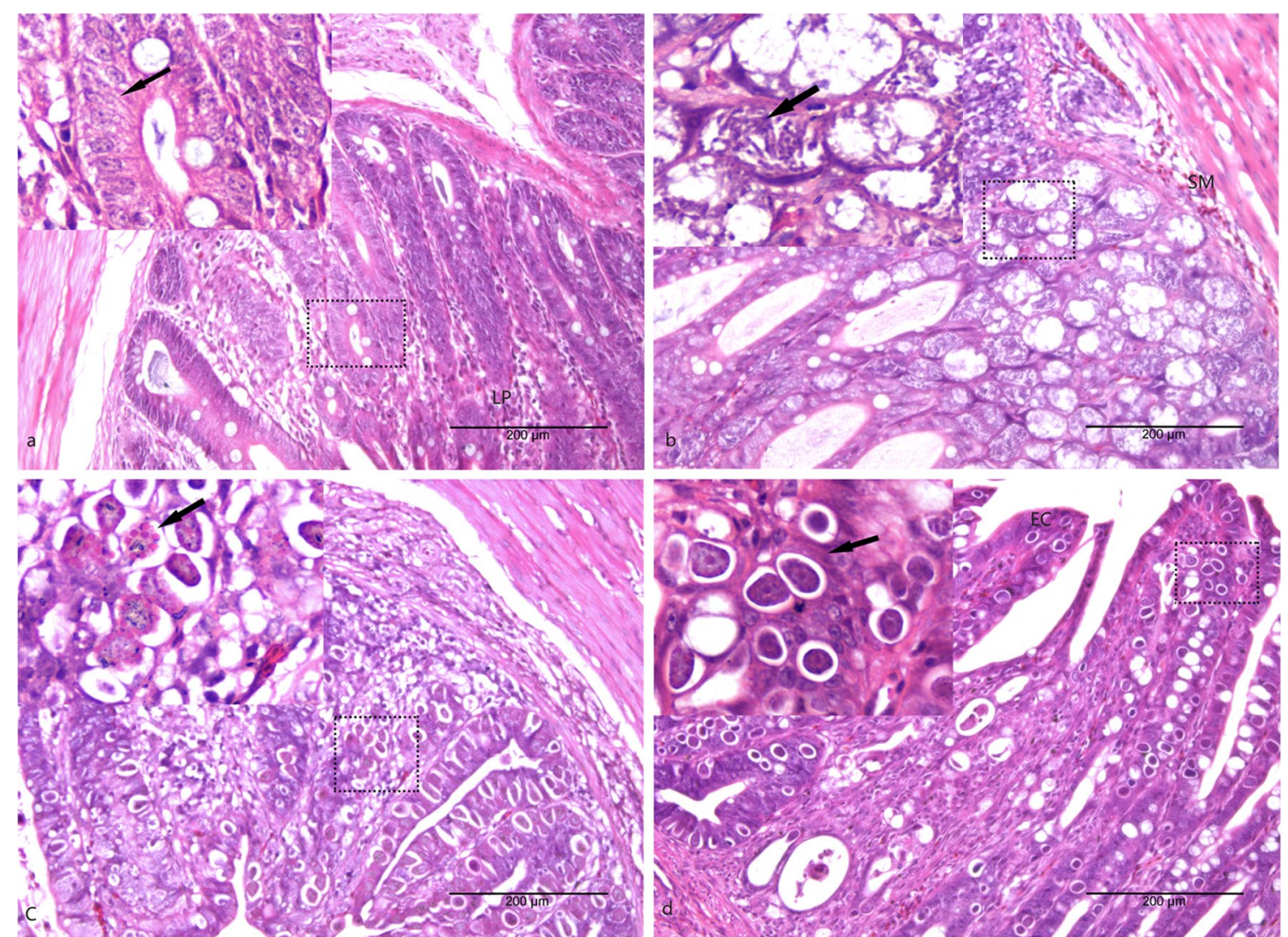

Fig. 1 Histopathological images of the cecum in AA broiler chickens from each group. a Section of the cecum from the uninfected chickens. $\mathbf{b}$ Section of the cecum from the $105 \mathrm{hpi}$ chickens in group M. c Section of the cecum from the $144 \mathrm{hpi}$ chickens in group G; visible gametocytes are indicated by arrows. $\mathbf{d}$ Section of the cecum from the $214 \mathrm{hpi}$ chickens in group O; visible oocysts are indicated by arrows. Abbreviations: EC, epithelial cell; LP, lamina propria; SM, submucosa. Magnifications: $\times 200$ and $\times 400$. Scale-bars: $200 \mu \mathrm{m}$

infected groups, but still higher than that of the control group. In conclusion, microbial shifts occurred in the cecum after E. tenella invasion. With the occurrence and development of invasion, microbiota was influenced at distinct time points (Fig. 3).

Principal coordinates analysis (PCoA) and NMDS ordination plots indicated that there was a small variation in the cecal constitution between group $C$ and group $\mathrm{M}$. The cecal microbial composition in groups $\mathrm{G}$ and $O$ had increased similarity, which means little variation occurred from $144 \mathrm{hpi}$ to 214 hpi. Furthermore, an alteration was observed in the microbial structure between group M and group G, from 105 hpi to 144 hpi. In combination with the alpha diversity results, the observed species and Shannon-Wiener indices showed a clear decline from $105 \mathrm{hpi}$ to $144 \mathrm{hpi}$, and the experimental data were consistent. Overall, the impacts of coccidial infection on the microbial community structure were roughly similar in agreement with the alpha diversity results in AA broiler chickens (Fig. 4).

\section{Bacterial taxa in the cecum after E. tenella infection}

To elucidate the effect of $E$. tenella infection on the composition of the cecal microbiota, we analyzed the bacteria at the phylum and genus levels to characterize the dynamics of microbial taxonomic distribution. At the phylum level, Firmicutes, Bacteroidetes, Tenericutes and Proteobacteria dominated the cecal microbial community in all four groups. In group $C$, the relative abundance of Firmicutes, Bacteroidetes, Tenericutes and Proteobacteria was $63.06 \%, 33.32 \%, 1.72 \%$ and $1.24 \%$, respectively. In group $M$, the relative abundance of Firmicutes and Tenericutes increased to $65.98 \%$ and $2.11 \%$, respectively, while the abundance of Bacteroidetes and Proteobacteria decreased to $29.20 \%$ and $1.14 \%$, respectively. In addition, we also observed Firmicutes (49.98\%), Bacteroidetes (43.82\%), Tenericutes $(0.46 \%)$ and Proteobacteria (5.26\%) in Group G, and Firmicutes (54.25\%), Bacteroidetes $(42.01 \%)$, Tenericutes (1.29\%) and Proteobacteria $(1.47 \%)$ in Group O. The results suggest that the dominant microbes in group $C$ and in group $M$ had increased 


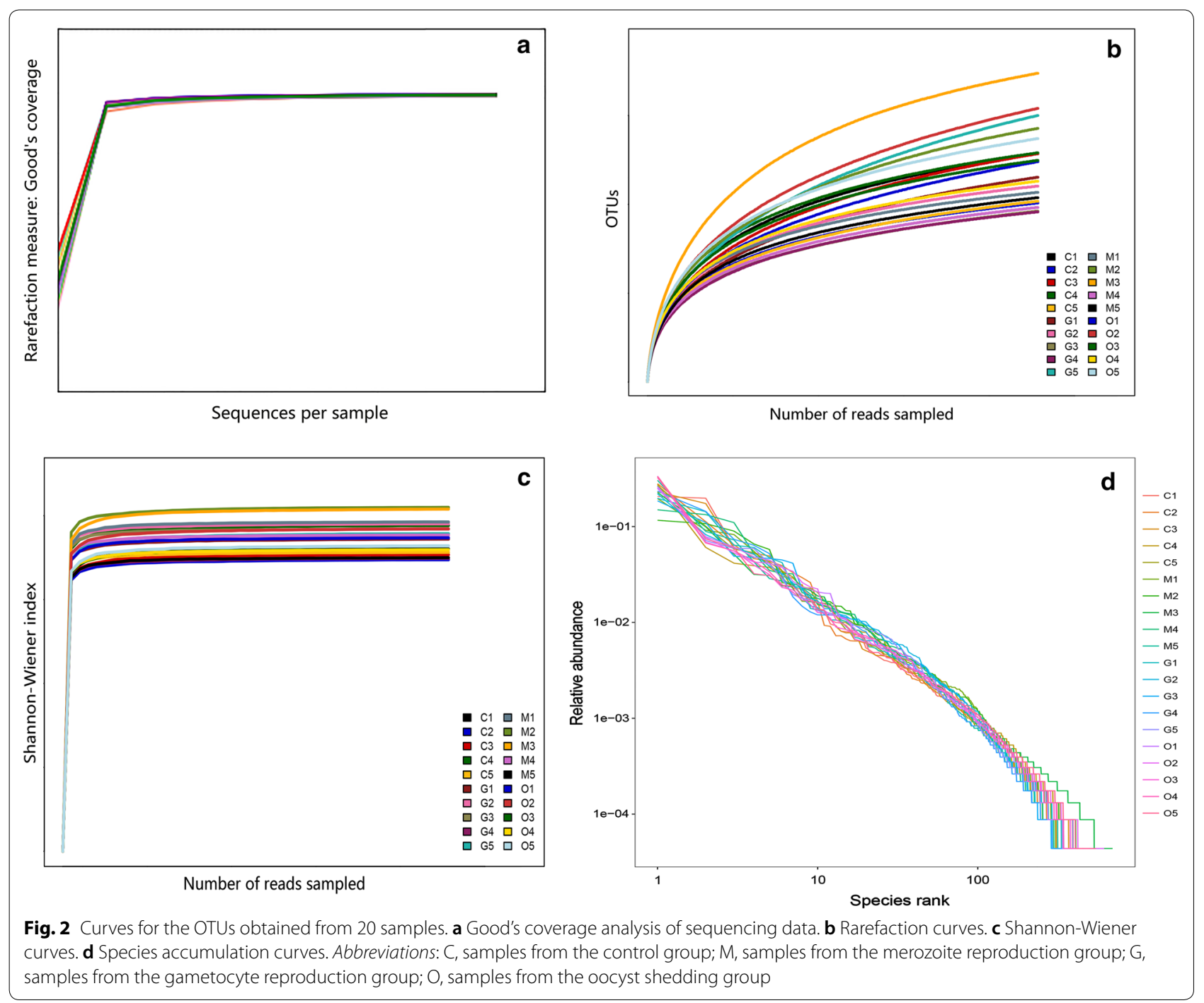

similarity, and group $\mathrm{G}$ and group $\mathrm{R}$ had increased similarity at the phylum level (Fig. 5).

We further compared the bacterial composition in the cecum at the genus level. A heatmap was also constructed based on the abundance profiles of the genera. Lactobacillus and Faecalibacterium belonging to the phylum Firmicutes, were important commensal microbiota. The relative abundance of Lactobacillus accounted for $18.02 \%, 17.86 \%, 11.87 \%$ and $14.59 \%$ of the population in groups $C, M, G$ and $O$, respectively, which showed a clear decline during the E. tenella infection period. The relative abundance of Faecalibacterium was 4.36\%, 2.51\%, $1.83 \%$ and $1.79 \%$ in groups $\mathrm{C}, \mathrm{M}, \mathrm{G}$ and $\mathrm{O}$, respectively. A linear decrease was also observed. Ruminococcaceae UCG-013 is a member of the Order Clostridiales, within the phylum Firmicutes. The relative abundance of Ruminococcaceae UCG-013 was outside of the abundance of the top 20 genera of all the samples. The heatmap showed that relative abundance of Ruminococcaceae UCG-013 decreased steadily with the development of the E. tenella infection (Fig. 6).

The heatmap also revealed that opportunistic pathogenic bacteria increased in relative abundance in the infected groups compared with the control group. Given the experimental data at different infection time points, we found that Enterococcus and Streptococcus, which both belong to the phylum Firmicutes, showed high abundance during E. tenella infection over time. The experimental results indicated that E. tenella infection impacts the microbiota composition in different phases of infection.

To fully understand the influence of E. tenella infection on gut microbiota, we performed a LEfSe analysis. The taxonomic cladogram and LDA score obtained from the LEfSe analysis confirmed and enabled the visualization of the impacts of infection (Figs. 7, 8). 

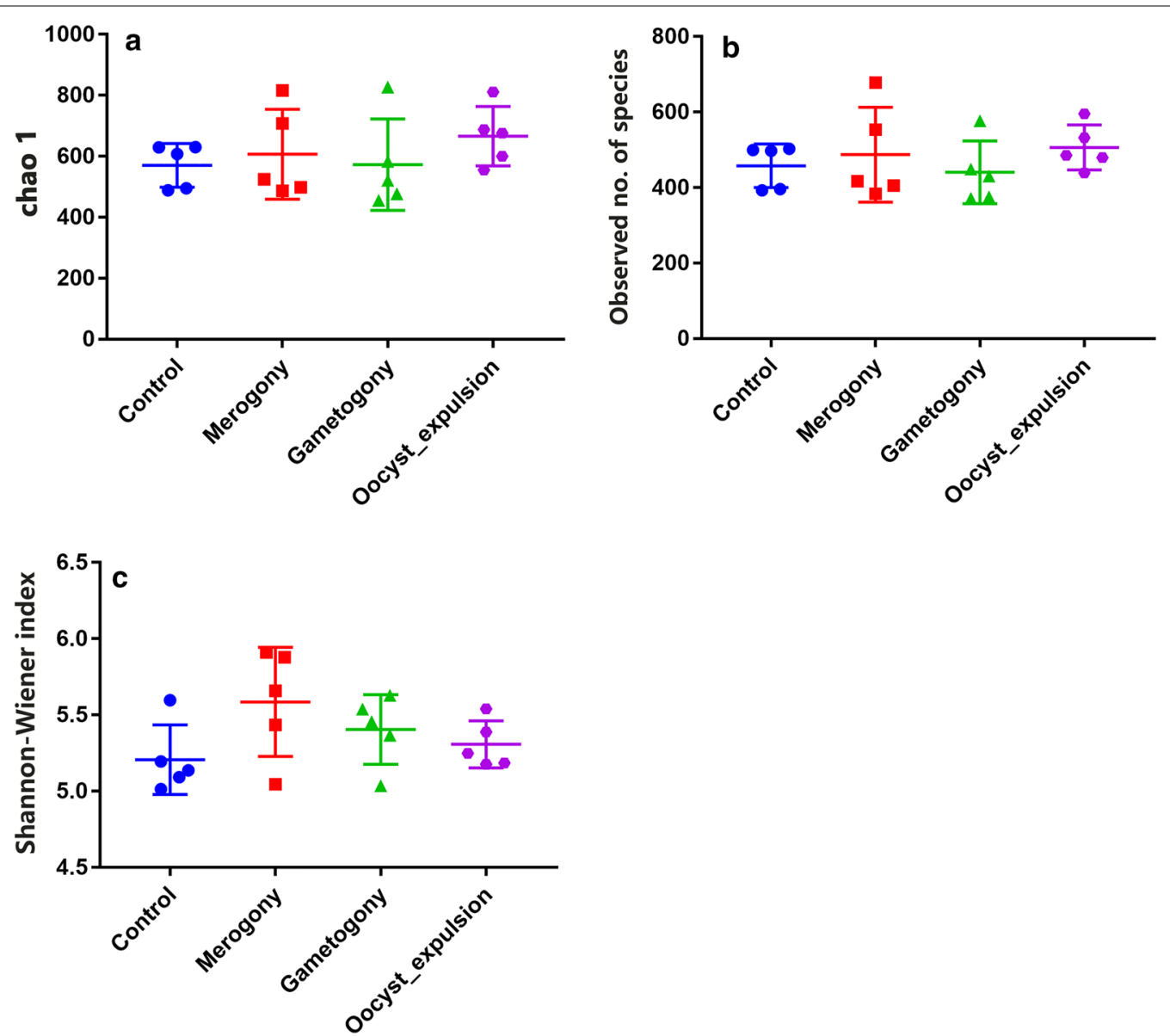

Fig. 3 Analysis of alpha-diversity in the four experimental groups. Chaol (a) and observed number of species (b) were used as richness estimators. Shannon-Wiener index (c) was used as a diversity estimator

The LEfSe analysis showed a significant decline in the abundances of the potentially beneficial bacteria Ruminococcaceae UCG-013 between the infected groups and the control group. Specifically, compared with the uninfected chickens, the amount of Ruminococcaceae UCG-013 was decreased by approximately 2 -fold at $144 \mathrm{hpi}$ in the infected chickens. The populations of Ruminococcaceae UCG-013 showed a mild increase at $214 \mathrm{hpi}$, which suggests the possibility of the recovery of the gut environment. We also found that the populations of Enterococcus spp., Streptococcus spp. and Bilophila spp. were increased over time in response to coccidia invasion. Co-occurrence network diagram of Firmicutes and Bacteroidetes was also conducted to show the relationship between some important members belonging to the top two dominant phylum (Figs. 9, 10, 11).

\section{Discussion}

Understanding the intestinal microbial composition and structure in chickens throughout the life-cycle of coccidial infection may help identify correlations between microbiota alterations and protozoan invasion over time, reveal potential biomarkers and may lead to the development of novel practical treatment methods [30]. In this study, we dissected the gut microbiota composition and structure in AA chickens at four time points during E. tenella infection utilizing $16 \mathrm{~S}$ rRNA gene sequencing. We found that commensal bacteria, such as Lactobacillus, Faecalibacterium, Ruminococcaceae UCG-013, Romboutsia and Shuttleworthia, declined in abundance after infection, whereas Enterococcus and Streptococcus were enriched in abundance in response to the infection. This finding is consistent with the microbiota constitution at the phylum level. All of these decreased 


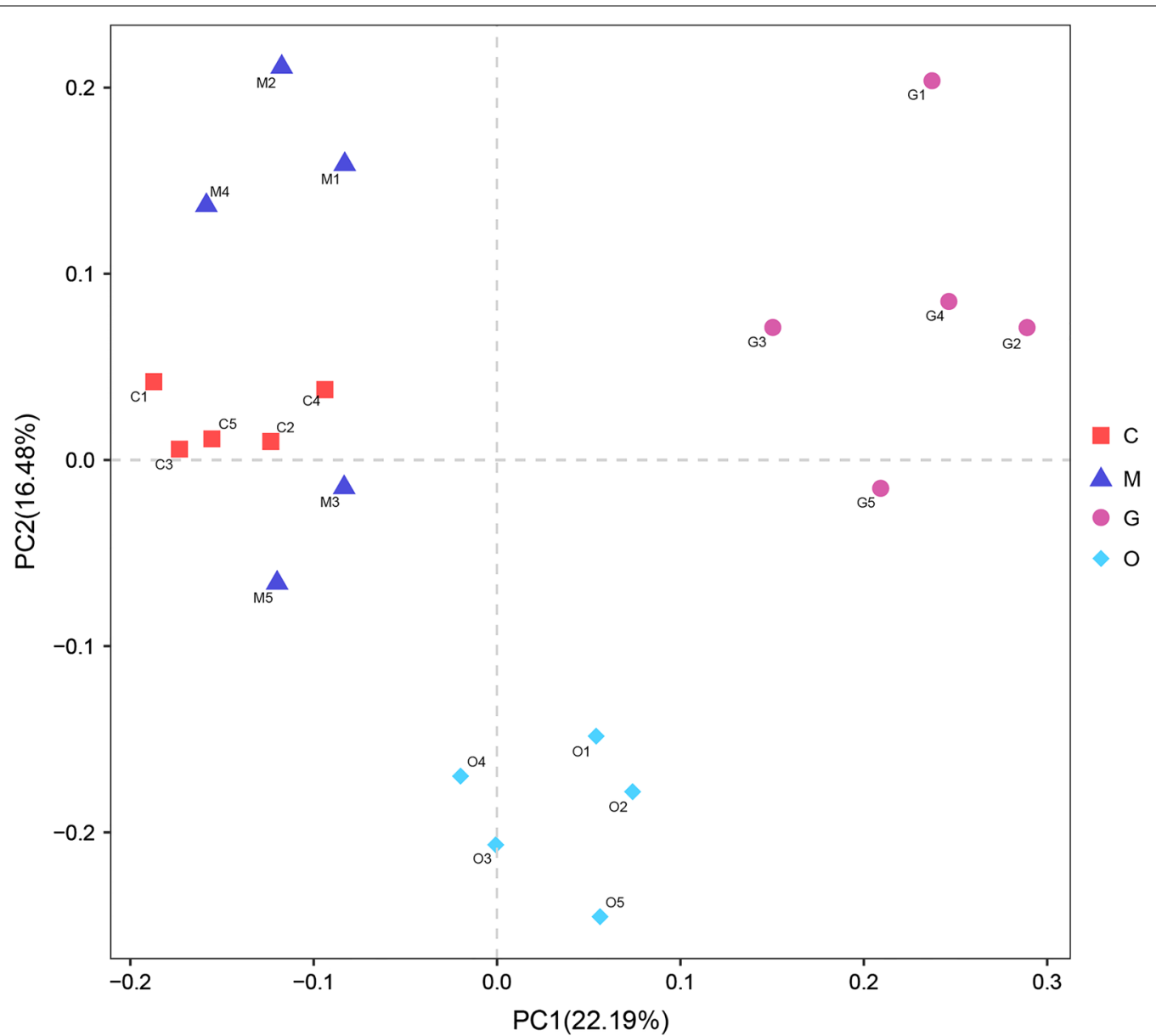

Fig. 4 Principal coordinates analysis of the structure of the gut microbiota. Abbreviations: $C$, samples from the control group; $M$, samples from the merozoite reproduction group; $\mathrm{G}$, samples from the gametocyte reproduction group; $\mathrm{O}$, samples from the oocyst shedding group

genera mentioned above belong to the phylum Firmicutes. In the merozoite reproduction period of infection, at the 105 hpi time point, the levels of Firmicutes barely changed, from $63.06 \%$ to $65.98 \%$. However, in the gametocyte reproduction period, at the $144 \mathrm{hpi}$ time point, the abundance levels of Firmicutes markedly declined from $65.98 \%$ to $49.98 \%$, which indicates the possibility of a decrease in the abundance of beneficial bacteria, and an increase in the emergence of opportunistic pathogenic bacteria. Our experimental results suggest that the late phase of the life-cycle of E. tenella infection in AA broilers impacted the homeostasis of the gut microbiota. The deficiency of defined resident microbiota may contribute to the pathogenicity of cecal coccidiosis.

Consistent with previous research, our study found that Firmicutes, Bacteroidetes, Tenericutes and Proteobacteria are the most common phyla in the chicken cecum. Firmicutes are the predominant phylum with the highest abundance [31]. The predominant phylum was mainly represented by Lachnospiraceae, Ruminococcaceae,
Lactobacillaceae, Peptostreptococcaceae and Clostridiaceae [32]. Some members of the phylum Firmicutes can inhibit the growth of opportunistic pathogens and some are known to be involved in the degradation of complex carbohydrates [33]. The abundance levels of Firmicutes were decreased in the late phase of infection in our study. Moreover, the genera Lactobacillus (Lactobacillaceae), Faecalibacterium (Clostridiaceae), Shuttleworthia (Lachnospiraceae), Ruminococcaceae UCG-013 (Ruminococcaceae) and Romboutsia (Peptostreptococcaceae) showed a coccidian-associated reduction in the life-cycle of asexual (105 hpi) and sexual (144 hpi) replication processes, with a particularly clear reduction in the sexual reproduction period. Lactobacillus, which is a beneficial commensal for humans and animals, has been studied and used in medicine and the food industry for years. It has been proven that the enrichment of Lactobacillus could generally improve the gastrointestinal tract environment, protect the gut from pathogens and promote intestinal mucosal immunity and energy extraction 

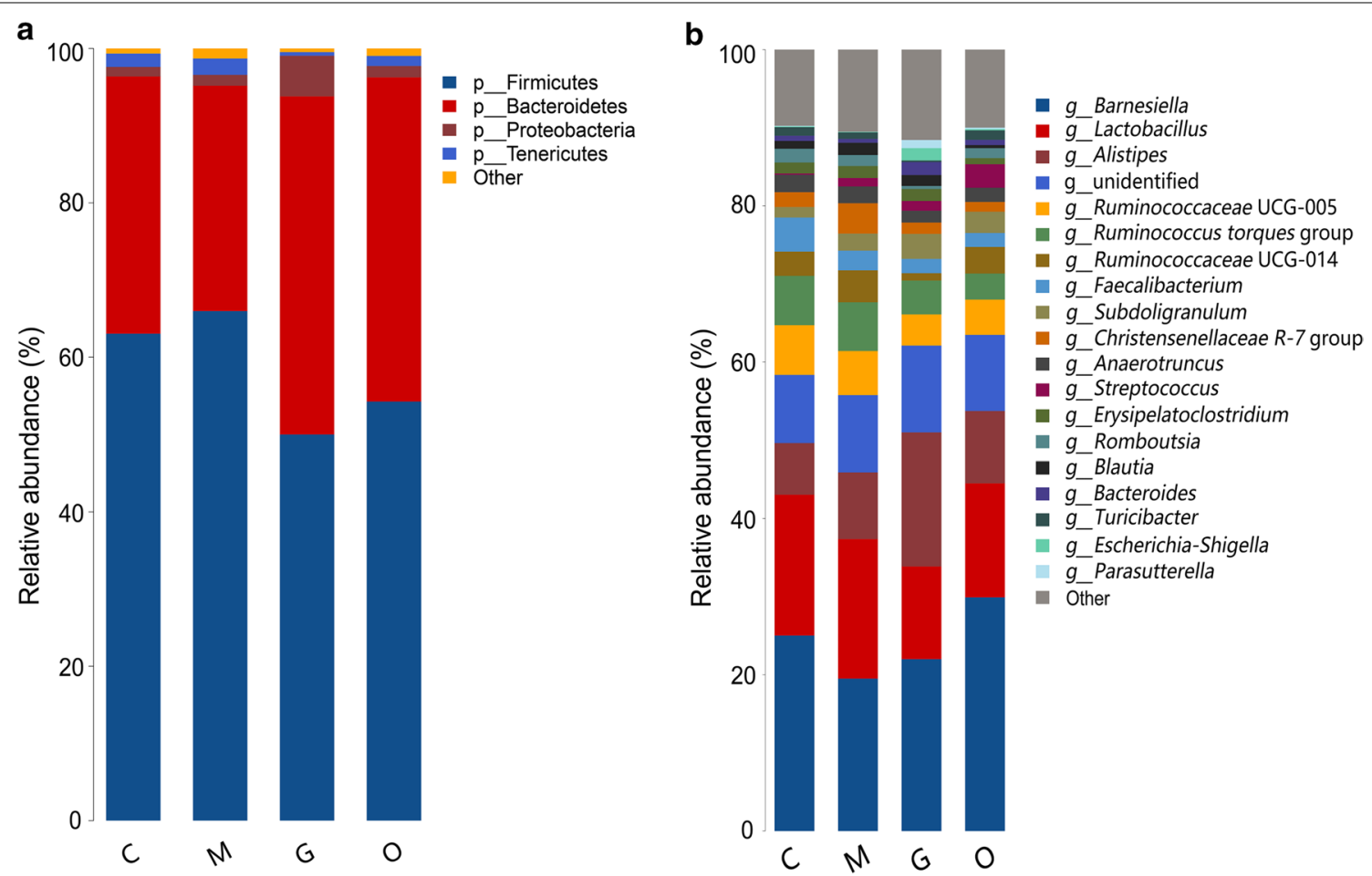

Fig. 5 The relative abundances of the cecal microbiota at the phylum (a) and genus (b) levels. The relative abundances of the gut bacteria presented here were calculated by averaging the data obtained from the five replicates within each group. Abbreviations: $\mathrm{C}$, control group; $\mathrm{M}$, merozoite reproduction group; $\mathrm{G}$, gametocyte reproduction group; $\mathrm{O}$, oocyst shedding group

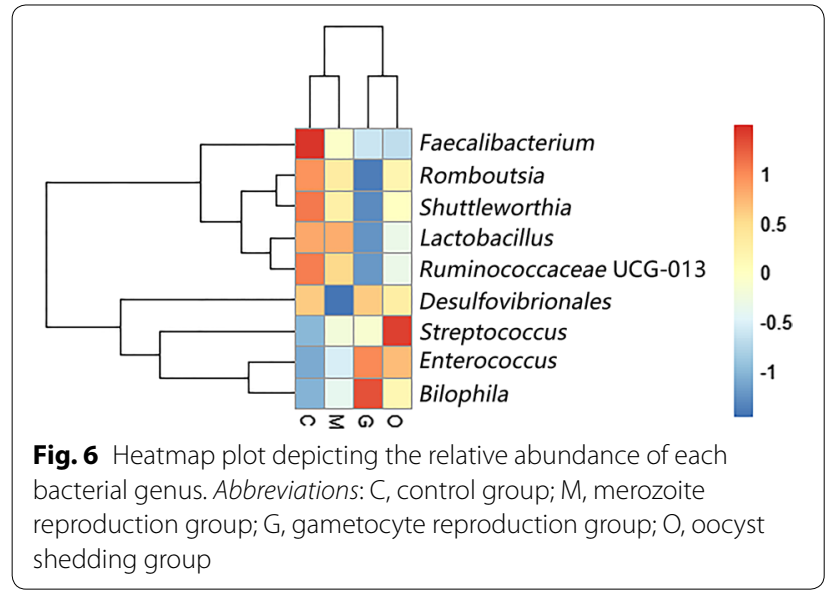

in the host [34, 35]. Faecalibacterium prausnitzii, which is the only species of Faecalibacterium, is regarded as a potentially beneficial microbe because it has been shown to have anti-inflammatory properties in humans and murine models [36, 37]. In addition, as a saccharolytic, butyrate-producing bacterium, Faecalibacterium is speculated to express enzymes favoring the production of butyrate to regulate the immune system, to reduce chronic inflammation, and also alleviate the pathogenicity of E. tenella infection [38, 39]. Ruminococcaceae are common intestinal microbiota that degrade complex carbohydrates [40]. Ruminococcaceae have carbohydrate-active enzymes, sugar transport mechanisms, and metabolic pathways for the degradation of complex plant materials [41]. A reduction in Ruminococcaceae UCG013 has been related to a disrupted carbon metabolism, which means body weight loss in chickens after E. tenella infection [42]. Romboutsia is a recently described bacterial genus that is usually associated with the health status of the gastrointestinal tract. The drastic reduction of this particular genus in intestinal mucosa may represent a potential microbial indicator of a disease condition. Romboutsia may play a crucial role in maintaining the health status of the host and could be a very valuable candidate biomarker of intestinal dysbiosis [43, 44]. Shuttleworthia has been demonstrated to have a relationship with carbohydrate and lipid metabolic pathways and thus contribute to weight gain and growth performance in broiler chickens [45]. However, opportunistic pathogenic bacteria of the genera Enterococcus and Streptococcus showed consistent increases in the infected AA chickens over time. Bisophila, which is a common non-pathogenic resident in the cecum of chickens, appears to be a consistent 


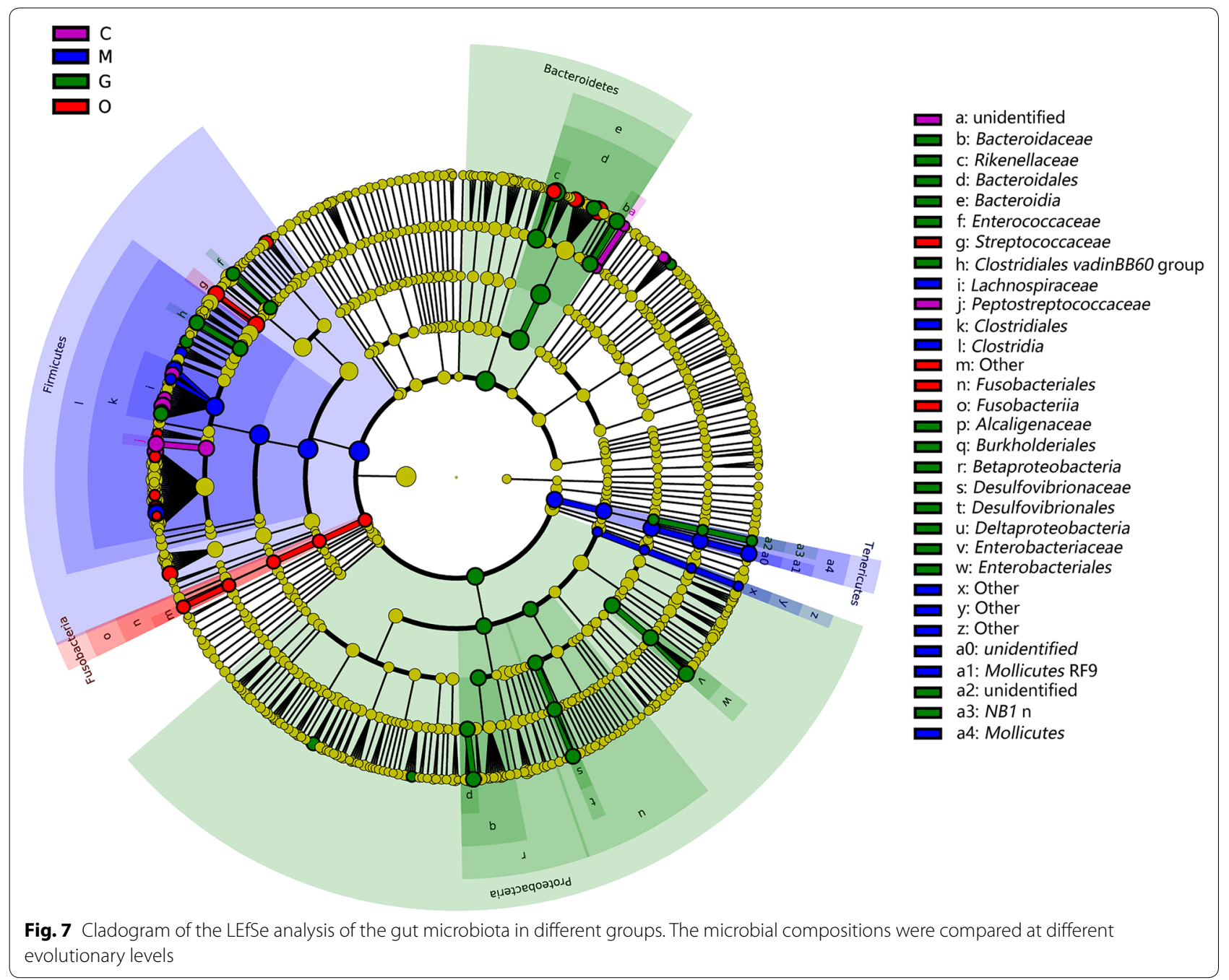

member of the anaerobic microbiota and may play a role in avian malabsorption syndromes [46].

LEfSe analysis indicated that Ruminococcaceae UCG013, Shuttleworthia and Romboutsia may act as candidate biomarkers of coccidiosis. Ruminococcaceae UCG-013 and Shuttleworthia both play a role in regulating the carbohydrate metabolic pathway, which may impact host digestion. In addition, from the co-occurrence network diagram, positive correlations were found to exist between Romboutsia (OTU13) and Lactobacillus (OTU2), Faecalibacterium (OTU534) and Ruminococcaceae UCG-013. Our findings highlight the possibility of using butyrate-producing bacteria such as Faecalibacterium, probiotics such as Lactobacillus, carbohydratedegrading bacteria such as Ruminococcaceae UCG-013 and Shuttleworthia, to reorganize gut microbiota to control coccidia infection.

The life-cycle of E. tenella includes asexual and sexual cycles. The asexual cycle consists of sporozoite and merozoite reproduction. The sexual cycle comprises microgamete reproduction. All reproductive processes occur inside the cecal epithelial cells [47]. In groups $\mathrm{C}$ and $\mathrm{M}$, at the early phase time point of $105 \mathrm{hpi}$, we found that the microbiota composition and structure were similar in the early phase, in agreement with the earlier study of Cryptosporidium infection, which is related to Eimeria [48]. In groups $\mathrm{M}$ and $\mathrm{G}$, from $105 \mathrm{hpi}$ to $144 \mathrm{hpi}$, beneficial bacteria decreased, and conditional pathogenic bacteria increased. Huang et al. [22] found similar performance both in the infected AA broiler and White Leghorn chickens at $120 \mathrm{hpi}$, where the relative abundance of Lactobacillus and Faecalibacterium decreased, and the relative abundance of the pathobionts Clostridium, Lysinibacillus and Escherichia increased. In our study the microbiota was greatly impacted, but no significant variation in alpha diversity was observed between the control group and the infected groups. From $105 \mathrm{hpi}$ to $144 \mathrm{hpi}$, E. tenella infection causes serious intestinal 


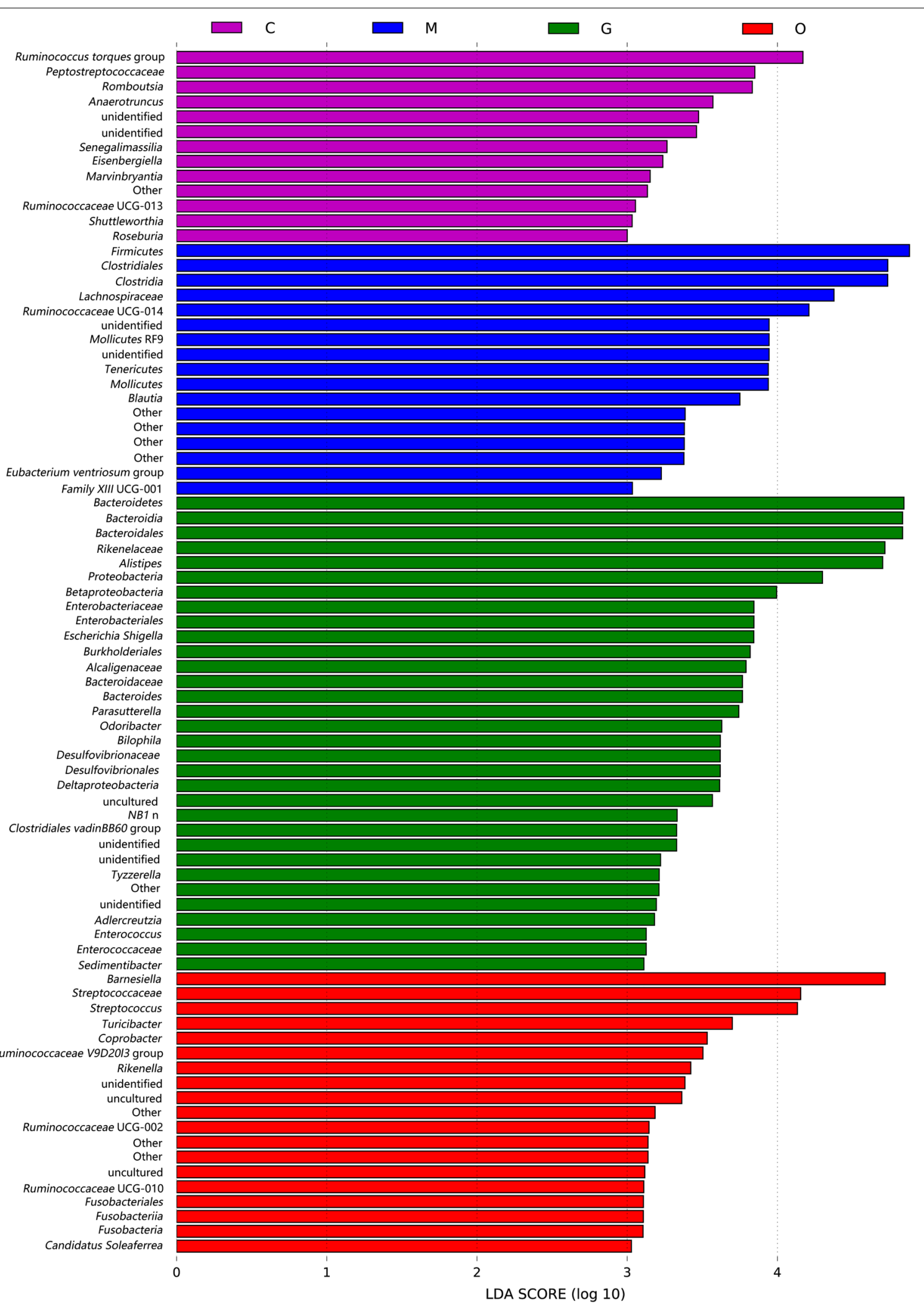

Fig. 8 LDA scores obtained from the LEfSe analysis of the gut microbiota in different groups. An LDA effect size of greater than 3 was used as a threshold for the LEfSe analysis. Abbreviations: C, control group; M, merozoite reproduction group; G, gametocyte reproduction group; O, oocyst shedding group 


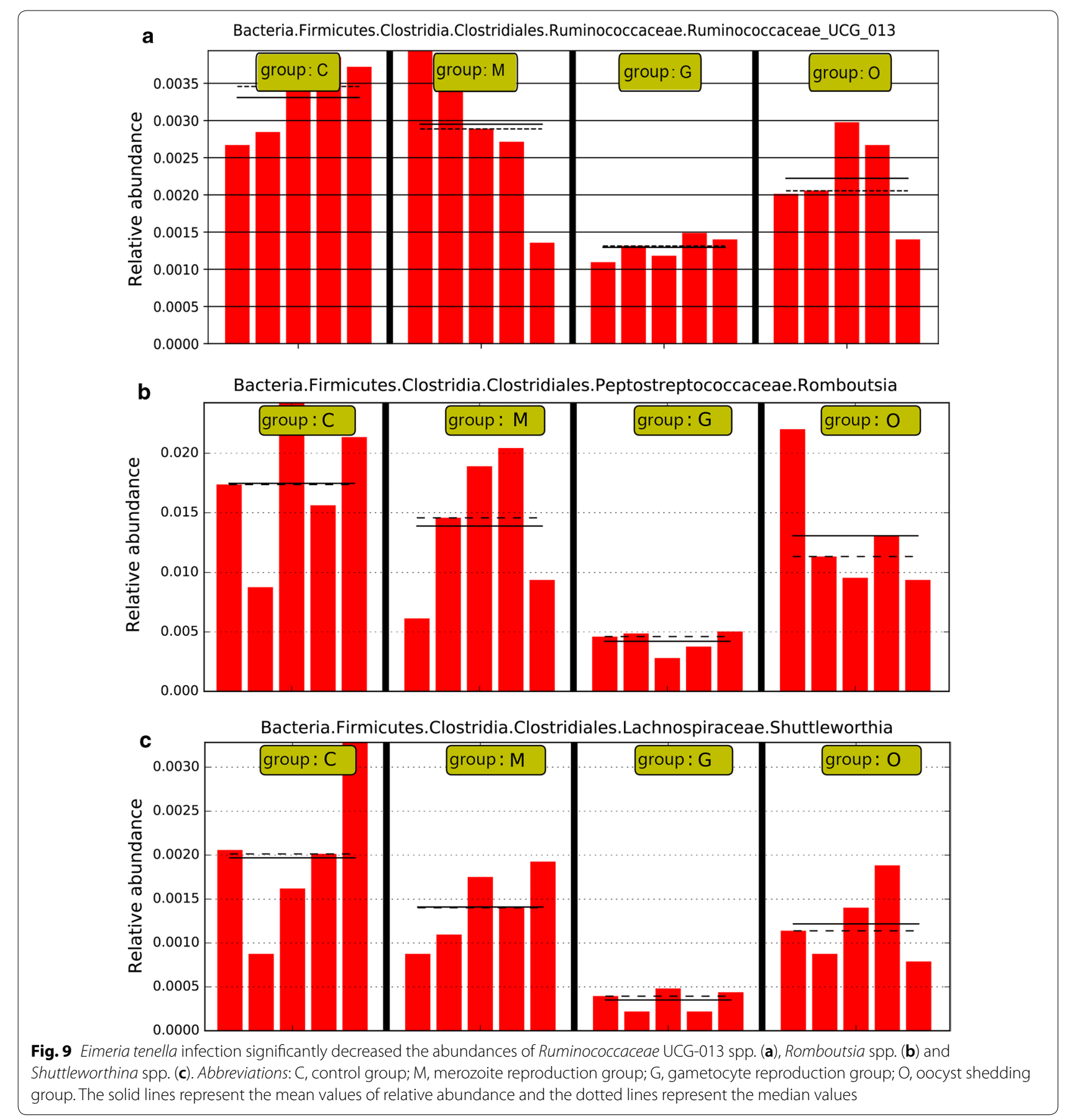

epithelial injuries that negatively impact the colonization and growth of resident bacteria, thus leading to decreased richness and diversity of cecal microbiota and increased risk of secondary infection [49]. MacDonald et al. [21] also reported that E. tenella infection induced no significant changes in the diversity of taxa in cecal microbial constitution, while the relative abundances of some genera such as Lactobacillus, Bifidobacterium and
Clostridium changed significantly between all uninfected samples and all infected samples. These authors also described some interesting findings that the birds which remained asymptomatic after $E$. tenella infection had increased levels of Lactobacillus and decreased levels of Bacteroides.

Lactobacillus-based probiotics have been demonstrated to exert anticoccidial properties on performance 

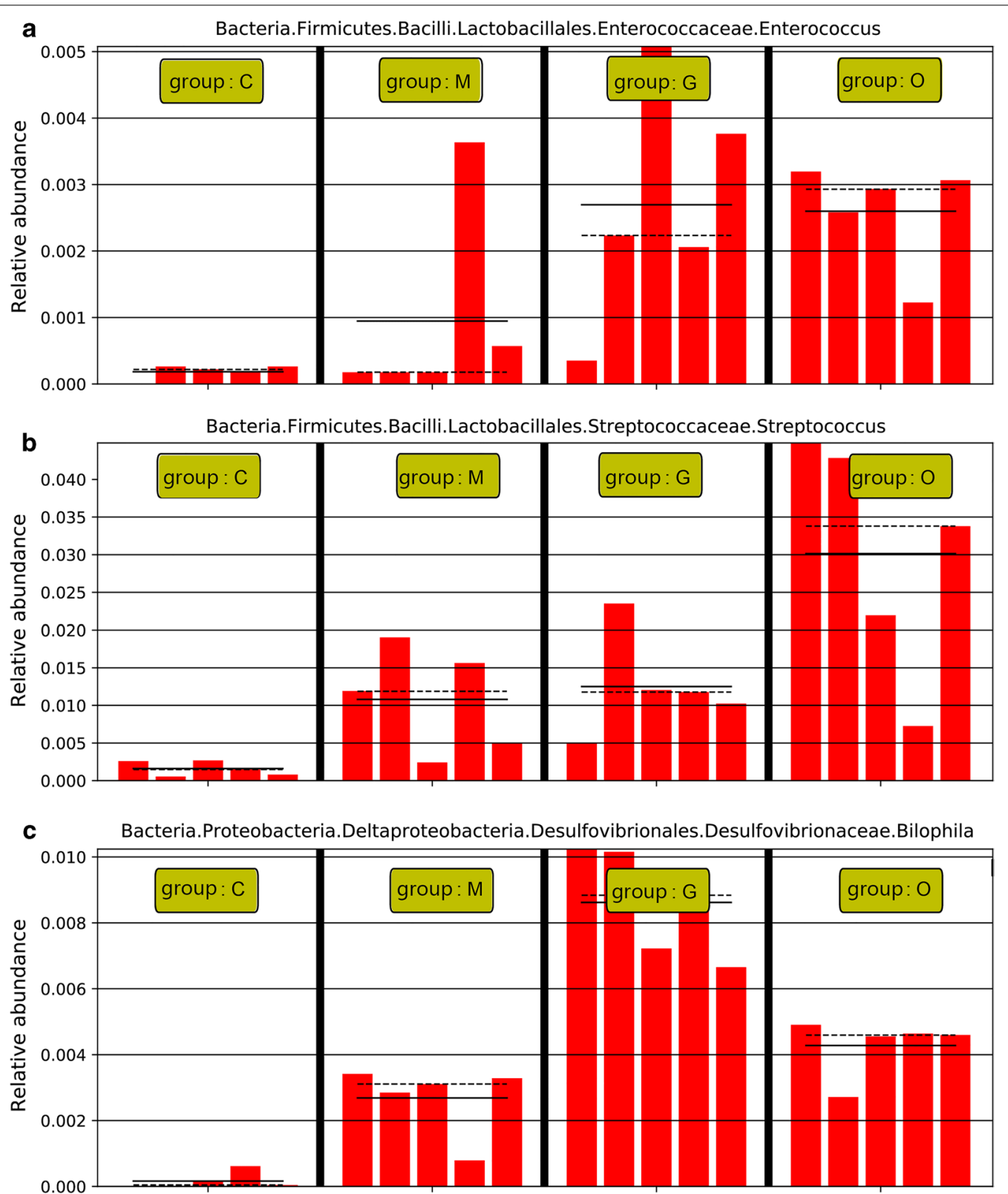

Fig. 10 Eimeria tenella infection significantly increased the abundance of Enterococcus spp. (a), Streptococcus spp. (b) and Bisophila spp. (c). Abbreviations: C, control group; M, merozoite reproduction group; G, gametocyte reproduction group; O, oocyst shedding group. The solid lines represent the mean values of relative abundance and the dotted lines represent the median values

parameters such as body weight gain, feed intake, feed conversion ratio, mortality, lesion score and oocysts output [50-52]. They were also shown to have a stimulating effect on the innate and adaptive immune system to stimulate intestinal intraepithelial lymphocyte subpopulations and cytokines including IFN- $\gamma$, IL-2, IL-1 $\beta$ and IL-6 [51-53]. To date, limited research has been conducted to study anticoccidial effects of Lactobacillus-based probiotics in vitro. Tierney et al. [54] determined the inhibition of $E$. tenella sporozoite invasion by Lactobacillus species for the first time, whereby three Lactobacillus strains and their secreted metabolites in the spent culture supernatant were confirmed to inhibit the parasite invasion into MDBK cells. Hessenberger et al. [18] established a fast and inexpensive in vitro tool to screen for probiotics with anticoccidial activity and showed that viable Lactobacillus reuteri \# 514 and Lactobacillus salivarius subsp. salivarius \# 505 


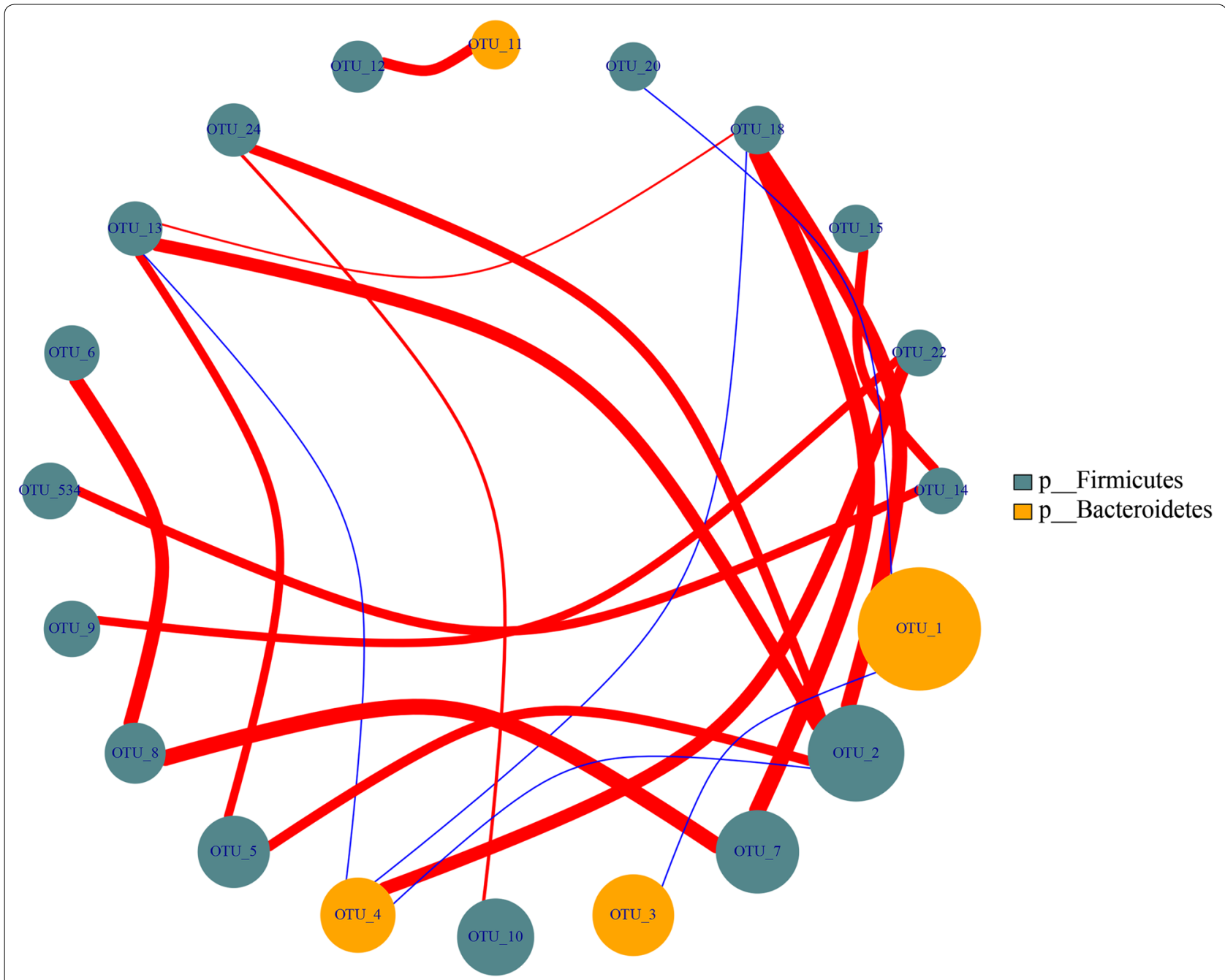

Fig. 11 Co-occurrence network diagram of Firmicutes and Bacteroidetes

could inhibit parasite invasion by more than $60 \%$ at the concentration of $10^{7} \mathrm{CFU} /$ well; however, the spent culture supernatant of both probiotics had no protection on parasite invasion. Considering the increasing resistance of parasites against anticoccidial drugs, Lactobacillus based-probiotics should be considered for the control of avian coccidiosis.

Our study focused on deciphering the changes of the gut microbiota composition after Eimeria infection and our findings indicate that dysbiosis of some resident flora may contribute to the pathogenicity of caecal coccidiosis. We did not consider the impact of the gut microbiota on coccidia. Therefore, in follow-up studies we plan to screen for Lactobacillus strains with anticoccidial activity in vitro first and subsequently investigate the potential protective use of suitable Lactobacillus strains-based probiotics in vivo against E. tenella; Faecalibacterium prausnitzii is also of interest in this context.

\section{Conclusions}

Altogether, the data in the present study demonstrate that gut microbiota shifts take place during the lifecycle of E. tenella infection. Infection with E. tenella impacts the cecal microbial composition and structure in AA broiler chickens. Non-pathogenic bacteria such as Lactobacillus, Faecalibacterium, Ruminococcaceae UCG-013, Romboutsia and Shuttleworthia, decreased in abundance. However, the opportunistic pathogens Enterococcus and Streptococcus were enriched in abundance. These findings further improve our current understanding of the influence of coccidia on microbiota during infection, indicate a correlation between time-related dysbiosis of gut flora and pathology and provides indications about the optimum time for therapeutic probiotics intervention. Our further research is to investigate the potential probiotics that have 


\section{anticoccidial effects and may aid in the development of novel control strategies against $E$. tenella infections.}

\author{
Abbreviations \\ AA broilers: Arbor Acre broilers; hpi.: hours post-infection; FLASH: fast length \\ adjustment of short reads; Qiime: quantitative insights into microbial ecology; \\ OTU: operational taxonomic units; PCA: principal components analysis; LDA: \\ linear discriminant analysis; LEfSe: linear discriminant analysis coupled with \\ the effect size; PCoA: principal coordinates analysis; MDBK: Madin-Darby \\ bovine kidney.
}

\section{Acknowledgements}

We thank Allwegene Technology Inc, Beijing, China, for sequencing and technical assistance.

\section{Authors' contributions}

GLY, QZ and CFW participated the design of this study. XYZ, GXZ and HBH contributed to the histopathology. HRL and CWS contributed to the DNA extraction, amplification and sequencing. HLC and WTY conducted the 165 rRNA sequencing results analysis. HLC, LPY, YLJ and JZW wrote and revised the manuscript. All authors read and approved the final manuscript.

\section{Funding}

This work was supported by the National Key Research and Development Programme of China (2017YFD0500400, 2017YFD0501200, 2017YFD0501000), the National Natural Science Foundation of China (31672528, 31602092) and the Science and Technology Development Programme of Jilin Province (20180201040NY, 20190301042NY).

\section{Availability of data and materials}

Data supporting the conclusions of this article are included within the article and its additional files. The raw reads of sequencing results were deposited into the NCBI Sequence Read Archive database under the Accession Number SRP184532.

\section{Ethics approval and consent to participate}

All animal experiments were conducted according to the guidelines established by the Association for Assessment and Acceleration of Laboratory Animal Care International. Animal experimental procedures were approved by the Ethics Committee of Jilin Agricultural University.

\section{Consent for publication}

Not applicable.

\section{Competing interests}

The authors declare that they have no competing interests.

Received: 27 March 2019 Accepted: 7 January 2020

Published online: 11 February 2020

\section{References}

1. Williams RB. Anticoccidial vaccines for broiler chickens: pathways to success. Avian Pathol. 2002;31:317-53.

2. Shirley MW, Smith AL, Tomley FM. The biology of avian Eimeria with an emphasis on their control by vaccination. Adv Parasitol. 2005;60:285.

3. Collier CT, Hofacre CL, Payne AM, Anderson DB, Kaiser P, et al. Coccidiainduced mucogenesis promotes the onset of necrotic enteritis by supporting Clostridium perfringens growth. Vet Immunol Immunopathol. 2008;122:104-15.

4. Qin ZR, Fukata T, Baba E, Arakawa A. Effect of Eimeria tenella infection on Salmonella enteritidis infection in chickens. Poult Sci. 1995;74:1-7.

5. MacDonald SE, van Diemen PM, Martineau H, Stevens MP, Tomley FM, et al. Impact of Eimeria tenella coinfection on Campylobacter jejuni colonization of the chicken. Infect Immun. 2019;87:e00772-00718.

6. Hume ME, Clemente-Hernandez S, Oviedo-Rondon EO. Effects of feed additives and mixed eimeria species infection on intestinal microbial ecology of broilers. Poult Sci. 2006:85:2106-11.
7. Oviedo-Rondon EO, Hume ME, Hernandez C, Clemente-Hernandez S. Intestinal microbial ecology of broilers vaccinated and challenged with mixed Eimeria species, and supplemented with essential oil blends. Poult Sci. 2006;85:854-60

8. Perez VG, Jacobs CM, Barnes J, Jenkins MC, Kuhlenschmidt MS, et al. Effect of corn distillers dried grains with solubles and Eimeria acervulina infection on growth performance and the intestinal microbiota of young chicks. Poult Sci. 2011;90:958-64.

9. Stanley D, Wu SB, Rodgers N, Swick RA, Moore RJ. Differential responses of cecal microbiota to fishmeal, Eimeria and Clostridium perfringens in a necrotic enteritis challenge model in chickens. PLOS ONE. 2014;9:e104739.

10. Peek HW, Landman WJ. Coccidiosis in poultry: anticoccidial products, vaccines and other prevention strategies. Vet Q. 2011;31:143-61.

11. Nicholson JK, Holmes E, Kinross J, Burcelin R, Gibson G, et al. Host-gut microbiota metabolic interactions. Science. 2012;336:1262-7.

12. Kelly D, Conway S, Aminov R. Commensal gut bacteria: mechanisms of immune modulation. Trends Immunol. 2005;26:326-33.

13. Giannenas I, Tsalie E, Triantafillou E, Hessenberger S, Teichmann K, et al. Assessment of probiotics supplementation via feed or water on the growth performance, intestinal morphology and microflora of chickens after experimental infection with Eimeria acervulina, Eimeria maxima and Eimeria tenella. Avian Pathol. 2014;43:209-16.

14. Giannenas I, Papadopoulos E, Tsalie E, Triantafillou E, Henikl S, et al. Assessment of dietary supplementation with probiotics on performance, intestinal morphology and microflora of chickens infected with Eimeria tenella. Vet Parasitol. 2012;188:31-40.

15. Abu-Akkada SS, Awad AM. Protective effects of probiotics and prebiotics on Eimeria tenella-infected broiler chickens. Pak Vet J. 2015;35:446-50.

16. Ritzi MM, Abdelrahman W, van-Heerden K, Mohnl M, Barrett NW, et al. Combination of probiotics and coccidiosis vaccine enhances protection against an Eimeria challenge. Vet Res. 2016;47:111.

17. Ritzi MM, Abdelrahman W, Mohnl M, Dalloul RA. Effects of probiotics and application methods on performance and response of broiler chickens to an Eimeria challenge. Poult Sci. 2014;93:2772-8.

18. Hessenberger S, Schatzmayr G, Teichmann K. In vitro inhibition of Eimeria tenella sporozoite invasion into host cells by probiotics. Vet Parasitol. 2016:229:93-8.

19. Biggs P, Parsons $C M$. The effects of several organic acids on growth performance, nutrient digestibilities, and cecal microbial populations in young chicks. Poult Sci. 2008:87:2581.

20. Zhao Y, Li B, Bai D, Huang J, Shiraigo W, et al. Comparison of fecal microbiota of Mongolian and thoroughbred horses by high-throughput sequencing of the V4 region of the $16 \mathrm{~S}$ rRNA gene. Asian-Australas J Anim Sci. 2016;29:1345-52.

21. MacDonald SE, Nolan MJ, Harman K, Boulton K, Hume DA, et al. Effects of Eimeria tenella infection on chicken caecal microbiome diversity, exploring variation associated with severity of pathology. PLOS ONE. 2017;12:e0184890.

22. Huang G, Tang X, Bi F, Hao Z, Han Z, et al. Eimeria tenella infection perturbs the chicken gut microbiota from the onset of oocyst shedding. Vet Parasitol. 2018;258:30-7.

23. Yang G, Wang C, Hao F, Zhao D, Zhang Y, et al. Studies on construction of a recombinant Eimeria tenella $\mathrm{SO}$ gene expressing Escherichia coli and its protective efficacy against homologous infection. Parasitol Int. 2010;59:517-23.

24. Yang G, Yao J, Yang W, Jiang Y, Du J, et al. Construction and immunological evaluation of recombinant Lactobacillus plantarum expressing $\mathrm{SO}$ of Eimeria tenella fusion DC-targeting peptide. Vet Parasitol. 2017;236:7-13.

25. Mori HI, Maruyama FU, Kato HI, et al. Design and experimental application of a novel non-degenerate universal primer set that amplifies prokaryotic $16 \mathrm{~S}$ rRNA genes with a low possibility to amplify eukaryotic rRNA genes. DNA Res. 2014;21:217-27.

26. Magoc T, Salzberg SL. FLASH: fast length adjustment of short reads to improve genome assemblies. Bioinformatics. 2011;27:2957-63.

27. Bolger AM, Lohse M, Usadel B. Trimmomatic: a flexible trimmer for Illumina sequence data. Bioinformatics. 2014;30:2114-20.

28. Caporaso JG, Kuczynski J, Stombaugh J, Bittinger K, Bushman FD, et al. QIIME allows analysis of high-throughput community sequencing data. Nat Methods. 2010;7:335-6. 
29. Segata N, Izard J, Waldron L, Gevers D, Miropolsky L, et al. Metagenomic biomarker discovery and explanation. Genome Biol. 2011;12:R60.

30. Quiroz-Castaneda RE, Dantan-Gonzalez E. Control of avian coccidiosis: future and present natural alternatives. Biomed Res Int. 2015;2015:430610.

31. Wei S, Morrison M, Yu Z. Bacterial census of poultry intestinal microbiome. Poult Sci. 2013;92:671-83.

32. Jiangrang L, Umelaalim I, Barry H, Charles H, Maurer JJ, et al. Diversity and succession of the intestinal bacterial community of the maturing broiler chicken. Appl Environ Microbiol. 2003;69:6816-24.

33. Kirsty B, Daniella D, Erin M, Gibson DL. Diet-induced dysbiosis of the intestinal microbiota and the effects on immunity and disease. Nutrients. 2012:4:1095-119.

34. Dicks LMT, Botes M. Probiotic lactic acid bacteria in the gastro-intestinal tract: health benefits, safety and mode of action. Benef Microbes. 2010;1:11-29.

35. Miquel S, Martin R, Rossi O, Bermudez-Humaran LG, Chatel JM, et al. Faecalibacterium prausnitzii and human intestinal health. Curr Opin Microbiol. 2013;16:255-61.

36. Willing BP, Dicksved J, Halfvarson J, Andersson AF, Lucio M, et al. A pyrosequencing study in twins shows that gastrointestinal microbial profiles vary with inflammatory bowel disease phenotypes. Gastroenterology. 2011;139:1844-54.

37. Sokol H, Pigneur B, Watterlot L, Lakhdari O, Bermudez-Humaran LG, et al. Faecalibacterium prausnitzii is an anti-inflammatory commensal bacterium identified by gut microbiota analysis of Crohn disease patients. Proc Natl Acad Sci USA. 2008;105:16731-6.

38. Zhou Z, Nie K, Huang Q, Li K, Sun Y, et al. Changes of cecal microflora in chickens following Eimeria tenella challenge and regulating effect of coated sodium butyrate. Exp Parasitol. 2017;177:73-81.

39. Machiels K, Joossens M, Sabino J, De Preter V, Arijs I, et al. A decrease of the butyrate-producing species Roseburia hominis and Faecalibacterium prausnitzii defines dysbiosis in patients with ulcerative colitis. Gut. 2014;63:1275-83.

40. Damer PB, Emily LC, Sarah EM, Venkatachalam T, Krishnendu K, et al. Population, genetic, and antigenic diversity of the apicomplexan Eimeria tenella and their relevance to vaccine development. Proc Natl Acad Sci USA. 2015;112:5343-50.

41. Biddle A, Stewart L, Blanchard J, Leschine S. Untangling the genetic basis of fibrolytic specialization by Lachnospiraceae and Ruminococcaceae in diverse gut communities. Diversity. 2013;5:627.

42. Hu R, Zeng F, Wu L, Wan X, Chen Y, et al. Fermented carrot juice attenuates type 2 diabetes by mediating gut microbiota in rats. Food Funct. 2019;10:2935-46.
43. Ricaboni D, Mailhe M, Khelaifia S, Raoult D, Million M. Romboutsia timonensis, a new species isolated from human gut. New Microbes New Infect. 2016;12:6-7.

44. Mangifesta M, Mancabelli L, Milani C, Gaiani F, de'Angelis N, et al. Mucosal microbiota of intestinal polyps reveals putative biomarkers of colorectal cancer. Sci Rep. 2018;8:13974.

45. Lee KC, Kil DY, Sul WJ. Cecal microbiome divergence of broiler chickens by sex and body weight. J Microbiol. 2017;55:939-45.

46. Mcorist S, Keller L, Mcorist A. Search for Lawsonia intracellularis and Bilophila wadsworthia in malabsorption-diseased chickens. Can J Vet Res. 2003;67:232.

47. Rose ME, Lawn AM, Millard BJ. The effect of immunity on the early events in the life-cycle of Eimeria tenella in the caecal mucosa of the chicken. Parasitology. 1984;88:199-210.

48. Refaat R, Kevin H, Enas D, Ahmed B, Giovanni W. Perturbation of the intestinal microbiota of mice infected with Cryptosporidium parvum. Int J Parasitol. 2015;45:567-73.

49. Kimura N, Mimura F, Nishida S, Kobayashi A. Studies on the relationship between intestinal flora and cecal coccidiosis in chicken. Poult Sci. 1976;55:1375

50. Bozkurt M, Aysul N, Kucukyilmaz K, Aypak S, Ege G, et al. Efficacy of infeed preparations of an anticoccidial, multienzyme, prebiotic, probiotic, and herbal essential oil mixture in healthy and Eimeria spp.-infected broilers. Poult Sci. 2014;93:389-99.

51. Chen CY, Chuang LT, Chiang YC, Li Lin C, Lien YY, et al. Use of a probiotic to ameliorate the growth rate and the inflammation of broiler chickens caused by Eimeria tenella infection. J Anim Res Nutr. 2016;1:10.

52. Dalloul RA, Lillehoj HS, Tamim NM, Shellem TA, Doerr JA. Induction of local protective immunity to Eimeria acervulina by a Lactobacillus-based probiotic. Comp Immunol Microbiol Infect Dis. 2005;28:351-61.

53. Dalloul RA, Lillehoj HS, Shellem TA, Doerr JA. Enhanced mucosa immunity against Eimeria acervulina in broilers fed a Lactobacillus-based probiotic. Poult Sci. 2003;82:62-6.

54. Tierney J, Gowing H, Van Sinderen D, Flynn S, Stanley L, et al. In vitro inhibition of Eimeria tenella invasion by indigenous chicken Lactobacillus species. Vet Parasitol. 2004;122:171-82.

\section{Publisher's Note}

Springer Nature remains neutral with regard to jurisdictional claims in published maps and institutional affiliations.
Ready to submit your research? Choose BMC and benefit from:

- fast, convenient online submission

- thorough peer review by experienced researchers in your field

- rapid publication on acceptance

- support for research data, including large and complex data types

- gold Open Access which fosters wider collaboration and increased citations

- maximum visibility for your research: over $100 \mathrm{M}$ website views per year

At BMC, research is always in progress.

Learn more biomedcentral.com/submissions 